\title{
APLICAÇÃO DOS BLOCOS INCOMPLETOS BALANCEADOS NA TEORIA DE RESPOSTA AO ITEM
}

\author{
Roberto M. Bekman \\ Eng $^{\circ}$ Eletricista pela Universidade de São Paulo - USP \\ Mestrando em Estatística pela Universidade de São Paulo - USP \\ Analista de Desenvolvimento na Fundação Carlos Chagas, São Paulo, SP
}

\begin{abstract}
Resumo
O artigo apresenta os Blocos Incompletos Balanceados (BIB) como uma possível solução para o problema de como se distribuir um grande número de questões (adequado para se obter informações amplas sobre o ensino) em cadernos de prova com poucas questões (adequados aos alunos). A forma de se distribuir as questões em Blocos Incompletos Balanceados é especialmente útil no uso conjunto com análises à luz da Teoria de Resposta ao Item (TRI) como forma de uniformizar a exposição das questões, visando a obtenção de resultados mais precisos. Neste sentido são apresentados vários exemplos de Blocos Incompletos Balanceados que atendem a diferentes relações entre o número de questões nos cadernos de prova dos alunos face ao total de questões do estudo.
\end{abstract}

Palavras-chave: Teoria da Resposta ao Item; blocos incompletos balanceados; construção de provas

\section{Resumen}

El artículo presenta los Bloques Incompletos Balanceados (BIB) como una posible solución para el problema de cómo distribuir un gran número de cuestiones (adecuados para que se obtengan informaciones amplias referentes a la enseñanza) en cuadernos de examenes con pocas cuestiones (adecuados para los alumnos). La forma de distribuir las cuestiones en Bloques Incompletos Balanceados es especialmente útil se usados en conjunto con el análisis a la luz de la Teoría de Respuesta al Iten (TRI), como forma de uniformizar la exposición de las cuestiones, de modo a obtener resultados más exactos. En este sentido, se presentan varios ejemplos de Bloques Incompletos Balanceados que atienden a diferentes relaciones entre el número de cuestiones en los cuadernos de examenes de los alumnos y el total de cuestiones del estudio.

Palabras-Ilave: Teoría de la respuesta al iten; bloques incompletos balanceados; elaboración de examenes. 


\begin{abstract}
The article presents the Balanced Incomplete Blocks (BIB) as a possible solution to the problem of how to distribute a great number of questions (appropriate to get broad information on education) in test booklets with few questions (appropriate to the students). The way of distributing the questions among Balanced Incomplete Blocks is especially useful when used together with analyses considering the Item Response Theory (IRT) as a way to make the display of the questions uniform so as to obtain more accurate results. In this sense several examples of Balanced Incomplete Blocks are presented that of different relations between the number of questions in the students' test booklets and the total questions of the study.
\end{abstract}

Keywords: Item Response Theory; balanced incomplete blocks (BIB); test construction 


\section{Introdução}

O modelo BIB (Blocos Incompletos Balanceados) foi idealizado há mais de 50 anos sem correlação direta com a montagem de cadernos de prova. A grosso modo, o BIB é um esquema otimizado para o rodízio de blocos com aplicações em diversas áreas, inclusive educação e agricultura. A necessidade do rodízio se justifica se pressupusermos que possuímos $b$ blocos e só podemos utilizar $k$ deles em cada conjunto. Isto é especialmente útil nos sistemas de avaliação quando desejamos obter informações amplas sobre o ensino, utilizando um grande número de itens, ao passo que precisamos limitar a quantidade de itens submetido a cada aluno num valor aceitável e adequado ao tempo de prova. A transposição do modelo BIB para a montagem de cadernos de prova pode ser resumida como abaixo:

a) distribuir um certo número $b$ de blocos de itens em diversos cadernos $c$ de forma que cada caderno não contenha a totalidade dos blocos. Estes cadernos incompletos são submetidos aos alunos que recebem, individualmente, um subconjunto do total de blocos de itens que está sendo aplicado na sala de aula, ou seja, cada aluno recebe apenas uma fração $f_{u}$ do total de blocos submetidos à sala de aula;

b) distribuir estes blocos de forma balanceada, onde

$\checkmark$ cada caderno contém o mesmo número de blocos $k_{i}$

$\checkmark$ cada bloco é utilizado o mesmo número de vezes $r$ dentro do conjunto total dos cadernos;

$\checkmark$ cada par de blocos é utilizado o mesmo número de vezes $\lambda$ dentro do conjunto total dos cadernos.

\section{Soluções BIB}

As soluções BIB só existem para determinadas combinações do número de cadernos $c$, número de blocos $b$ e número de blocos em cada caderno $k$. Para desenvolver as soluções BIB utilizaremos as seguintes convenções: 
$>c=$ Número de cadernos;

$>b=$ Número de blocos;

$>k$ = Número de blocos em cada caderno;

$>r=$ Número de repetições de cada bloco no conjunto total dos cadernos;

$\lambda=$ Número de repetições de cada par de blocos no conjunto total dos cadernos;

$>f_{u}=$ Fator de utilização.

Assim, para que haja um esquema solução BIB, é necessário que as relações abaixo sejam satisfeitas Uma combinação de $c, b, r, k$, $\lambda$ que satisfaça as relações não garante, entretanto, a existência de um esquema solução BIB. FEDERER (1955) e VAJDA (1967) abordam o problema de existência ou não de solução para os esquemas BIB. Para o restante do artigo vamos nos ater aos casos que admitem solução.

$$
\begin{aligned}
& \text { (i) } \quad c=\frac{r \cdot b}{k} \text {; } \\
& \text { (ii) } \lambda=\frac{r \cdot(k-1)}{(b-1)}, c, b, r, k, \lambda \in \mathrm{N} \text {. }
\end{aligned}
$$

Adicionalmente, um esquema solução poderá ou não ser classificado como BIB-Espiral. Para ser classificado como tal as seguintes propriedades devem ser satisfeitas, além de (i) e (ii):

(iii) $c=n \cdot b, n \in \mathrm{N}$;

(iv) os blocos devem estar distribuídos em espiral no conjunto dos cadernos de prova.

Para nós é importante estabelecer a razão entre o número de (blocos de) itens nos cadernos de prova dos alunos face ao total de (blocos de) itens do estudo, denominado por fator de utilização. De (i) concluímos que:

$$
\text { (v) } f_{u}=\frac{k}{b}=\frac{r}{c}=\text { Fator de utilização }
$$


De (v) verificamos que o fator de utilização também pode ser entendido como a proporção dos alunos que são submetidos a determinado bloco dentre o total de alunos.

\section{Exemplos}

A seguir serão apresentadas várias soluções para os esquemas BIB e, preferencialmente, BIB-Espiral. Os exemplos selecionados abrangem uma grande gama para o fator de utilização permitindo selecionar o esquema mais adequado à amplitude do estudo.

\subsection{Exemplo 1}

\begin{tabular}{|c|c|c|}
\hline Caderno & Bloco1 & Bloco2 \\
\hline 1 & 1 & 2 \\
\hline 2 & 2 & 3 \\
\hline 3 & 3 & 1 \\
\hline $\begin{array}{l}=3 r \\
=3 \lambda\end{array}$ & $\begin{array}{l}=2 \\
=1\end{array}$ & \\
\hline$=2 f_{u}$ & $=2 / 3$ & 0,67 \\
\hline
\end{tabular}

Neste exemplo:

$>$ Esquema BIB-Espiral.

> Foram elaborados 3 blocos (provas) diferentes.

> Foram compostos 3 conjuntos (cadernos de prova) com 2 dos 3 blocos.

> Cada bloco tem uma tiragem de $2 / 3$ do número total de conjuntos a serem impressos.

> Cada bloco é submetido a 2/3 do número total de alunos.

> Cada aluno é submetido a 2/3 dos blocos em sala de aula.

\subsection{Exemplo 2}

\section{Caderno Bloco1 Bloco2 Bloco3}




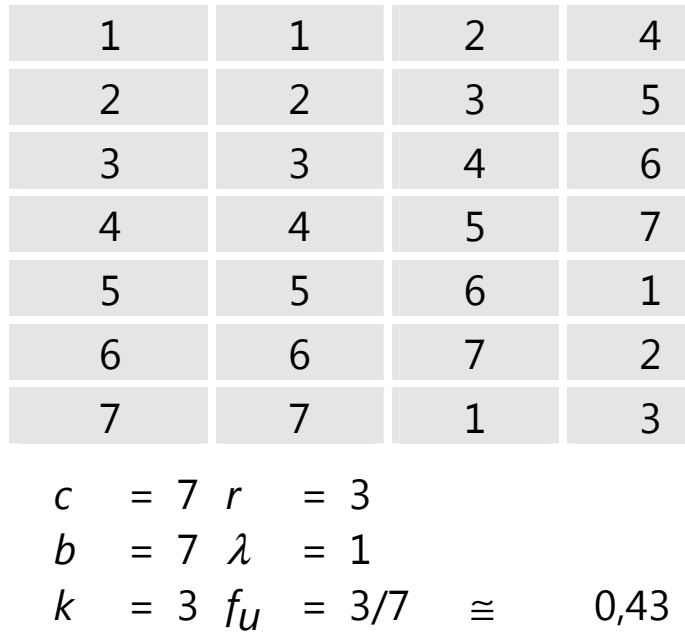

Neste exemplo:

$>$ Esquema BIB-Espiral.

$>$ Foram elaborados 7 blocos (provas) diferentes.

$>$ Foram compostos 7 conjuntos (cadernos de prova) com 3 dos 7 blocos.

> Cada bloco tem uma tiragem de 3/7 do número total de conjuntos a serem impressos.

> Cada bloco é submetido a 3/7 do número total de alunos.

> Cada aluno é submetido a 3/7 dos blocos em sala de aula.

\subsection{Exemplo 3}

\begin{tabular}{|c|c|c|}
\hline Caderno & Bloco1 & Bloco2 \\
\hline 1 & 1 & 2 \\
\hline 2 & 2 & 3 \\
\hline 3 & 3 & 4 \\
\hline 4 & 4 & 5 \\
\hline 5 & 5 & 1 \\
\hline 6 & 1 & 3 \\
\hline 7 & 2 & 4 \\
\hline 8 & 3 & 5 \\
\hline
\end{tabular}




\begin{tabular}{rl|l|l|} 
& 9 & 4 & 1 \\
& $10 \quad$ & 5 & 2 \\
$c=$ & $10 r=4$ \\
$b=$ & $5 \lambda=1$ \\
$k=$ & $2 f_{U}=2 / 5=0,40$
\end{tabular}

Neste exemplo:

$>$ Esquema BIB-Espiral.

> Foram elaborados 5 blocos (provas) diferentes.

> Foram compostos 10 conjuntos (cadernos de prova) com 2 dos 5 blocos.

> Cada bloco tem uma tiragem de 2/5 do número total de conjuntos a serem impressos.

> Cada bloco é submetido a 2/5 do número total de alunos.

> Cada aluno é submetido a 2/5 dos blocos em sala de aula.

\subsection{Exemplo 4}

\begin{tabular}{|c|c|c|c|c|}
\hline Caderno & Bloco1 & Bloco2 & Bloco3 & Bloco4 \\
\hline 1 & 1 & 2 & 4 & 10 \\
\hline 2 & 2 & 3 & 5 & 11 \\
\hline 3 & 3 & 4 & 6 & 12 \\
\hline 4 & 4 & 5 & 7 & 13 \\
\hline 5 & 5 & 6 & 8 & 1 \\
\hline 6 & 6 & 7 & 9 & 2 \\
\hline 7 & 7 & 8 & 10 & 3 \\
\hline 8 & 8 & 9 & 11 & 4 \\
\hline 9 & 9 & 10 & 12 & 5 \\
\hline 10 & 10 & 11 & 13 & 6 \\
\hline 11 & 11 & 12 & 1 & 7 \\
\hline 12 & 12 & 13 & 2 & 8 \\
\hline 13 & 13 & 1 & 3 & 9 \\
\hline
\end{tabular}




$$
\begin{aligned}
& c=13 r=4 \\
& b=13 \lambda=1 \\
& k=4 f_{u}=4 / 13 \cong 0,31
\end{aligned}
$$

Neste exemplo:

$>$ Esquema BIB-Espiral.

> Foram elaborados 13 blocos (provas) diferentes.

> Foram compostos 13 conjuntos (cadernos de prova) com 4 dos 13 blocos.

> Cada bloco tem uma tiragem de 4/13 do número total de conjuntos a serem impressos.

> Cada bloco é submetido a 4/13 do número total de alunos.

$>$ Cada aluno é submetido a 4/13 dos blocos em sala de aula. 


\subsection{Exemplo 5}

\begin{tabular}{|c|c|c|c|c|}
\hline Caderno & Bloco1 & Bloco2 & Bloco3 & Bloco4 \\
\hline 1 & 1 & 2 & 3 & 4 \\
\hline 2 & 1 & 5 & 9 & 13 \\
\hline 3 & 1 & 6 & 11 & 16 \\
\hline 4 & 1 & 7 & 12 & 14 \\
\hline 5 & 1 & 8 & 10 & 15 \\
\hline 6 & 2 & 5 & 12 & 15 \\
\hline 7 & 2 & 6 & 10 & 14 \\
\hline 8 & 2 & 7 & 9 & 16 \\
\hline 9 & 2 & 8 & 11 & 13 \\
\hline 10 & 3 & 5 & 10 & 16 \\
\hline 11 & 3 & 6 & 12 & 13 \\
\hline 12 & 3 & 8 & 9 & 14 \\
\hline 13 & 3 & 7 & 11 & 15 \\
\hline 14 & 4 & 5 & 11 & 14 \\
\hline 15 & 4 & 6 & 9 & 15 \\
\hline 16 & 4 & 7 & 10 & 13 \\
\hline 17 & 4 & 8 & 12 & 16 \\
\hline 18 & 5 & 6 & 7 & 8 \\
\hline 19 & 9 & 10 & 11 & 12 \\
\hline 20 & 13 & 14 & 15 & 16 \\
\hline$C$ & $=20 r$ & $=5$ & \multirow{3}{*}{\multicolumn{2}{|c|}{0,25}} \\
\hline$b$ & $=16 \lambda$ & $=1$ & & \\
\hline$k$ & $=4 f_{u}$ & $=1 / 4$ & & \\
\hline
\end{tabular}

Neste exemplo:

$>$ Esquema BIB não Espiral.

$>$ Foram elaborados 16 blocos (provas) diferentes.

> Foram compostos 20 conjuntos (cadernos de prova) com 4 dos 16 blocos.

> Cada bloco tem uma tiragem de 1/4 do número total de conjuntos a serem impressos. 
> Cada bloco é submetido a 1/4 do número total de alunos.

$>$ Cada aluno é submetido a $1 / 4$ dos blocos em sala de aula.

\subsection{Exemplo 6}

\begin{tabular}{|c|c|c|}
\hline Caderno & Bloco1 & Bloco2 \\
\hline 1 & 1 & 2 \\
\hline 2 & 2 & 3 \\
\hline 3 & 3 & 4 \\
\hline 4 & 4 & 5 \\
\hline 5 & 5 & 6 \\
\hline 6 & 6 & 7 \\
\hline 7 & 7 & 1 \\
\hline 8 & 1 & 3 \\
\hline 9 & 2 & 4 \\
\hline 10 & 3 & 5 \\
\hline 11 & 4 & 6 \\
\hline 12 & 5 & 7 \\
\hline 13 & 6 & 1 \\
\hline 14 & 7 & 2 \\
\hline 15 & 1 & 4 \\
\hline 16 & 2 & 5 \\
\hline 17 & 3 & 6 \\
\hline 18 & 4 & 7 \\
\hline 19 & 5 & 1 \\
\hline 20 & 6 & 2 \\
\hline 21 & 7 & 3 \\
\hline$=21 r$ & $=6$ & \\
\hline$b=7 \lambda$ & $=1$ & \\
\hline$k=2 f_{u}$ & $=2 / 7$ & 0,29 \\
\hline
\end{tabular}

Neste exemplo:

$>$ Esquema BIB-Espiral. 
> Foram elaborados 7 blocos (provas) diferentes.

> Foram compostos 21 conjuntos (cadernos de prova) com 2 dos 7 blocos.

> Cada bloco tem uma tiragem de 2/7 do número total de conjuntos a serem impressos.

> Cada bloco é submetido a 2/7 do número total de alunos.

> Cada aluno é submetido a 2/7 dos blocos em sala de aula.

\subsection{Exemplo 7}

\begin{tabular}{|c|c|c|c|c|c|}
\hline Caderno & Bloco1 & Bloco2 & Bloco3 & Bloco4 & Bloco5 \\
\hline 1 & 1 & 2 & 5 & 15 & 17 \\
\hline 2 & 2 & 3 & 6 & 16 & 18 \\
\hline 3 & 3 & 4 & 7 & 17 & 19 \\
\hline 4 & 4 & 5 & 8 & 18 & 20 \\
\hline 5 & 5 & 6 & 9 & 19 & 21 \\
\hline 6 & 6 & 7 & 10 & 20 & 1 \\
\hline 7 & 7 & 8 & 11 & 21 & 2 \\
\hline 8 & 8 & 9 & 12 & 1 & 3 \\
\hline 9 & 9 & 10 & 13 & 2 & 4 \\
\hline 10 & 10 & 11 & 14 & 3 & 5 \\
\hline 11 & 11 & 12 & 15 & 4 & 6 \\
\hline 12 & 12 & 13 & 16 & 5 & 7 \\
\hline 13 & 13 & 14 & 17 & 6 & 8 \\
\hline 14 & 14 & 15 & 18 & 7 & 9 \\
\hline 15 & 15 & 16 & 19 & 8 & 10 \\
\hline 16 & 16 & 17 & 20 & 9 & 11 \\
\hline 17 & 17 & 18 & 21 & 10 & 12 \\
\hline 18 & 18 & 19 & 1 & 11 & 13 \\
\hline 19 & 19 & 20 & 2 & 12 & 14 \\
\hline 20 & 20 & 21 & 3 & 13 & 15 \\
\hline 21 & 21 & 1 & 4 & 14 & 16 \\
\hline
\end{tabular}

Estudos em Avaliação Educacional, n² 24, jul-dez/2001 


$$
\begin{aligned}
& c=21 r=5 \\
& b=21 \lambda=1 \\
& k=5 f_{u}=5 / 21 \cong 0,24
\end{aligned}
$$

Neste exemplo:

$>$ Esquema BIB-Espiral.

> Foram elaborados 21 blocos (provas) diferentes.

> Foram compostos 21 conjuntos (cadernos de prova) com 5 dos 21 blocos.

> Cada bloco tem uma tiragem de 5/21 do número total de conjuntos a serem impressos.

> Cada bloco é submetido a $5 / 21$ do número total de alunos.

$>$ Cada aluno é submetido a 5/21 dos blocos em sala de aula.

\section{$3.8 \quad$ Exemplo 8}

\begin{tabular}{|c|c|c|c|}
\hline Caderno & Bloco1 & Bloco2 & Bloco3 \\
\hline 1 & 1 & 2 & 5 \\
\hline 2 & 2 & 3 & 6 \\
\hline 3 & 3 & 4 & 7 \\
\hline 4 & 4 & 5 & 8 \\
\hline 5 & 5 & 6 & 9 \\
\hline 6 & 6 & 7 & 10 \\
\hline 7 & 7 & 8 & 11 \\
\hline 8 & 8 & 9 & 12 \\
\hline 9 & 9 & 10 & 13 \\
\hline 10 & 10 & 11 & 1 \\
\hline 11 & 11 & 12 & 2 \\
\hline 12 & 12 & 13 & 3 \\
\hline 13 & 13 & 1 & 4 \\
\hline 14 & 1 & 6 & 8 \\
\hline 15 & 2 & 7 & 9 \\
\hline 16 & 3 & 8 & 10 \\
\hline 17 & 4 & 9 & 11 \\
\hline
\end{tabular}




\begin{tabular}{|c|c|c|c|}
\hline 18 & 5 & 10 & 12 \\
\hline 19 & 6 & 11 & 13 \\
\hline 20 & 7 & 12 & 1 \\
\hline 21 & 8 & 13 & 2 \\
\hline 22 & 9 & 1 & 3 \\
\hline 23 & 10 & 2 & 4 \\
\hline 24 & 11 & 3 & 5 \\
\hline 25 & 12 & 4 & 6 \\
\hline 26 & 13 & 5 & 7 \\
\hline
\end{tabular}

$$
\begin{aligned}
c & =26 r=6 \\
b & =13 \lambda=1 \\
k & =3 f_{u}=3 / 13 \cong 0,23
\end{aligned}
$$

Neste exemplo:

$>$ Esquema BIB-Espiral.

> Foram elaborados 13 blocos (provas) diferentes.

> Foram compostos 26 conjuntos (cadernos de prova) com 3 dos 13 blocos.

$>$ Cada bloco tem uma tiragem de 3/13 do número total de conjuntos a serem impressos.

$>$ Cada bloco é submetido a 3/13 do número total de alunos.

$>$ Cada aluno é submetido a 3/13 dos blocos em sala de aula.

\section{Perguntas e Respostas Freqüentes}

P1) Quando utilizar os esquemas BIB?

R1) Basicamente quando for desejável ou necessário distribuir uma grande quantidade de itens em vários cadernos de prova com poucos itens cada.

P2) Por que utilizar os esquemas BIB? 
R2) Porque a combinação dos esquemas BIB com uma logística adequada na distribuição dos cadernos de prova proporcionará os melhores resultados dentro do contexto da pergunta R1: Os itens serão respondidos aproximadamente pelo mesmo número de alunos, os alunos receberão cadernos com o mesmo número de blocos, os cadernos não conterão blocos repetidos e cada par de blocos será submetido ao mesmo número de alunos. As conseqüências imediatas desta metodologia serão melhores estimativas dos parâmetros dos itens e da habilidade dos alunos.

P3) É possível utilizar um determinado item em mais de um bloco BIB?

R3) Não. Sabemos pelas propriedades do modelo BIB que cada par de blocos se repete $\lambda$ vezes dentro do conjunto dos cadernos de prova. Assumindo que não podemos repetir nenhum item num mesmo caderno de prova concluímos que $\lambda$ precisa ser igual a 1 e que cada item não pode ser utilizado em mais de um bloco.

P4) Como escolher o esquema BIB mais adequado?

R4) Idealmente gostaríamos de colocar o maior número de itens em sala de aula mantendo-se uma quantidade aceitável de itens nos cadernos de prova (menor fator de utilização possível). Na prática devemos conciliar o ideal com as seguintes limitações:

> para estimar os parâmetros dos itens é necessário que cada um deles seja respondido por um número mínimo de alunos. Se desejamos realizar análises segundo a Teoria de Resposta ao Item é usual impor que cada item seja respondido por pelo menos 200 alunos, assim $f_{U}>200 /$ estimativa do número de alunos presentes;

$>$ não queremos que cada caderno contenha muitos blocos ki 
$>$ não queremos montar muitos blocos $b$;

$>$ não queremos colecionar muitos cadernos $c$.

Selecionamos então o esquema mais enxuto que tenha um fator de utilização $\left(f_{u}\right)$ apropriado.

P5) Entre um Esquema BIB e um Esquema BIB-Espiral, equivalentes entre si, qual devemos escolher?

R5) A princípio não haveria distinção entre os dois pois ambos atendem aos mesmos princípios. Na prática devemos dar preferência aos esquemas BIB-Espiral. Um esquema BIB-Espiral garante que cada bloco aparece o mesmo número de vezes em cada uma das posições no caderno. Observe que no Exemplo 5 (não Espiral) os blocos 1, 2, 3 e 4 se concentram no início dos cadernos e os blocos 13, 14, 15 e 16 no final dos cadernos. Se pressupusermos que o desempenho do aluno varie de uma forma determinística entre o início e o fim da prova, concluiremos que devemos utilizar os esquemas BIBEspiral afim de evitar distorções nas estimativas dos parâmetros dos itens. Adicionalmente os esquemas BIB permitem uma racionalização no processo de coleção dos cadernos de prova que pode ser importante para tiragens elevadas.

P6) Qual a tiragem de cada bloco $T_{b}$ se a tiragem total é $T_{t}$ ?

R6) A tiragem de cada bloco é $T_{b}=f_{u} \cdot T_{t}$.

P7) Quantos itens $I_{t}$ são necessários no total para que os alunos recebam cadernos de prova com $I_{C}$ itens cada?

R7) Da forma que foi definido o fator de utilização sai diretamente que $I_{t}=I_{c} / f_{u}$, com o número de itens no bloco $I_{b}=I_{c} / \mathrm{k}$ sendo um número inteiro. Para tornar os resultados mais claros tomemos como exemplo o SAEB (Sistema de Avaliação do Ensino Básico - MEC/INEP/DAEB). No SAEB foi utilizado o 
esquema BIB-Espiral de 26 cadernos equivalente ao exemplo 8. Cada bloco foi composto com 13 itens para que os cadernos ficassem com $I_{c}=I_{b} \cdot k=13=13 \times 3=39$ itens ( $\left.\cong 40\right)$, um número razoável para ser respondido pelos alunos. Por conseguinte $\mathrm{o}$ número total de itens (em cada série $\mathrm{e}$ disciplina) foi $I_{t}=I_{c} / f_{u}$ ou $I_{t}=I_{b} \cdot k / f_{u}=I_{c} \cdot b=13 \times 13=$ 169 itens. Se outro esquema fosse utilizado, como o do exemplo 6, cada caderno poderia ser montado com 20 itens (40 itens no caderno) e $I_{t}=40 \div 2 / 7=140$ itens no total. Ou seja, não temos liberdade total para definir cada um dos parâmetros mas, na maioria dos casos, conseguimos caminhar para uma solução que compatibilize nossas necessidades.

\section{Conclusão}

Os Blocos Incompletos Balanceados são muito úteis nos processos de avaliação pois permitem que conciliemos o grande número de itens necessários ao estudo da performance da sala de aula com o pequeno número de itens que podemos submeter aos alunos individualmente. Os Blocos Incompletos Balanceados têm a propriedade de distribuir os itens de forma uniforme entre o conjunto dos alunos desde que observados alguns cuidados durante a aplicação dos testes. A combinação da Teoria de Resposta ao Item com os Blocos Incompletos Balanceados nos permite obter informações precisas sobre a performance do aluno e da sala de aula simultaneamente. $O$ uso conjunto destas mesmas técnicas também pode ser utilizado para que um banco de itens testados e calibrados cresça num ritmo muito maior e consistente do que seria possível com outras metodologias. 


\section{Apêndice A}

Tabela com as combinações de cadernos c (até 200), blocos $b$ (até 200) e número de blocos por caderno $k$ (até 50 ) passíveis de admitir solução para os esquemas de Blocos Incompletos Balanceados (BIB), considerando $\lambda=1$ :

\begin{tabular}{|c|c|c|c|c|}
\hline $\begin{array}{l}\text { Número de } \\
\text { Cadernos } \\
\text { (c) }\end{array}$ & $\begin{array}{l}\text { Número } \\
\text { de } \\
\text { Blocos } \\
\text { (b) }\end{array}$ & $\begin{array}{l}\text { Número de } \\
\text { Blocos por } \\
\text { Caderno (k) }\end{array}$ & $\begin{array}{l}\text { Número de } \\
\text { Repetições de } \\
\text { cada Bloco (r) }\end{array}$ & $\begin{array}{c}\text { Fator de } \\
\text { utilização }\left(f_{\mathbf{u}}\right)\end{array}$ \\
\hline 3 & 3 & 2 & 2 & 0.67 \\
\hline 6 & 4 & 2 & 3 & 0.50 \\
\hline 7 & 7 & 3 & 3 & 0.43 \\
\hline 8 & 16 & 6 & 3 & 0.38 \\
\hline 10 & 5 & 2 & 4 & 0.40 \\
\hline 12 & 9 & 3 & 4 & 0.33 \\
\hline 13 & 13 & 4 & 4 & 0.31 \\
\hline 14 & 21 & 6 & 4 & 0.29 \\
\hline 15 & 6 & 2 & 5 & 0.33 \\
\hline 15 & 45 & 12 & 4 & 0.27 \\
\hline 20 & 16 & 4 & 5 & 0.25 \\
\hline 21 & 7 & 2 & 6 & 0.29 \\
\hline 21 & 21 & 5 & 5 & 0.24 \\
\hline 23 & 46 & 10 & 5 & 0.22 \\
\hline 24 & 96 & 20 & 5 & 0.21 \\
\hline 26 & 13 & 3 & 6 & 0.23 \\
\hline 28 & 8 & 2 & 7 & 0.25 \\
\hline 30 & 25 & 5 & 6 & 0.20 \\
\hline 31 & 31 & 6 & 6 & 0.19 \\
\hline 33 & 55 & 10 & 6 & 0.18 \\
\hline 34 & 85 & 15 & 6 & 0.18 \\
\hline 35 & 15 & 3 & 7 & 0.20 \\
\hline 35 & 175 & 30 & 6 & 0.17 \\
\hline 36 & 9 & 2 & 8 & 0.22 \\
\hline
\end{tabular}




\begin{tabular}{|l|l|l|l|l|}
\hline 42 & 36 & 6 & 7 & 0.17 \\
\hline 43 & 43 & 7 & 7 & 0.16 \\
\hline 45 & 10 & 2 & 9 & 0.20 \\
\hline 46 & 92 & 14 & 7 & 0.15 \\
\hline
\end{tabular}

\begin{tabular}{|c|c|c|c|c|}
\hline $\begin{array}{l}\text { Número de } \\
\text { Cadernos } \\
\text { (c) }\end{array}$ & $\begin{array}{l}\text { Número } \\
\text { de } \\
\text { Blocos } \\
\text { (b) }\end{array}$ & $\begin{array}{l}\text { Número de } \\
\text { Blocos por } \\
\text { Caderno (k) }\end{array}$ & $\begin{array}{l}\text { Número de } \\
\text { Repetições de } \\
\text { cada Bloco (r) }\end{array}$ & $\begin{array}{c}\text { Fator de } \\
\text { utilização }\left(\mathbf{f}_{\mathbf{u}}\right)\end{array}$ \\
\hline 47 & 141 & 21 & 7 & 0.15 \\
\hline 50 & 25 & 4 & 8 & 0.16 \\
\hline 55 & 11 & 2 & 10 & 0.18 \\
\hline 56 & 49 & 7 & 8 & 0.14 \\
\hline 57 & 19 & 3 & 9 & 0.16 \\
\hline 57 & 57 & 8 & 8 & 0.14 \\
\hline 60 & 105 & 14 & 8 & 0.13 \\
\hline 63 & 28 & 4 & 9 & 0.14 \\
\hline 66 & 12 & 2 & 11 & 0.17 \\
\hline 69 & 46 & 6 & 9 & 0.13 \\
\hline 70 & 21 & 3 & 10 & 0.14 \\
\hline 72 & 64 & 8 & 9 & 0.12 \\
\hline 73 & 73 & 9 & 9 & 0.12 \\
\hline 75 & 100 & 12 & 9 & 0.12 \\
\hline 77 & 154 & 18 & 9 & 0.12 \\
\hline 78 & 13 & 2 & 12 & 0.15 \\
\hline 82 & 41 & 5 & 10 & 0.12 \\
\hline 85 & 51 & 6 & 10 & 0.12 \\
\hline 90 & 81 & 9 & 10 & 0.11 \\
\hline 91 & 14 & 2 & 13 & 0.14 \\
\hline 91 & 91 & 10 & 10 & 0.11 \\
\hline 94 & 141 & 15 & 10 & 0.11 \\
\hline 95 & 171 & 18 & 10 & 0.11 \\
\hline 99 & 45 & 5 & 11 & 0.11 \\
\hline 100 & 25 & 3 & 12 & 0.12 \\
\hline
\end{tabular}




\begin{tabular}{|r|r|r|l|l|}
\hline 105 & 15 & 2 & 14 & 0.13 \\
\hline 110 & 100 & 10 & 11 & 0.10 \\
\hline 111 & 37 & 4 & 12 & 0.11 \\
\hline 111 & 111 & 11 & 11 & 0.10 \\
\hline 117 & 27 & 3 & 13 & 0.11 \\
\hline 120 & 16 & 2 & 15 & 0.12 \\
\hline 122 & 61 & 6 & 12 & 0.10 \\
\hline 130 & 40 & 4 & 13 & 0.10 \\
\hline 132 & 121 & 11 & 12 & 0.09 \\
\hline
\end{tabular}

\begin{tabular}{|c|c|c|c|c|}
\hline $\begin{array}{l}\text { Número de } \\
\text { Cadernos } \\
\text { (c) }\end{array}$ & $\begin{array}{l}\text { Número } \\
\text { de } \\
\text { Blocos } \\
\text { (b) }\end{array}$ & $\begin{array}{l}\text { Número de } \\
\text { Blocos por } \\
\text { Caderno (k) }\end{array}$ & $\begin{array}{l}\text { Número de } \\
\text { Repetições de } \\
\text { cada Bloco (r) }\end{array}$ & $\begin{array}{c}\text { Fator de } \\
\text { utilização }\left(\mathbf{f}_{\mathbf{u}}\right)\end{array}$ \\
\hline 133 & 133 & 12 & 12 & 0.09 \\
\hline 136 & 17 & 2 & 16 & 0.12 \\
\hline 143 & 66 & 6 & 13 & 0.09 \\
\hline 153 & 18 & 2 & 17 & 0.11 \\
\hline 155 & 31 & 3 & 15 & 0.10 \\
\hline 156 & 144 & 12 & 13 & 0.08 \\
\hline 157 & 157 & 13 & 13 & 0.08 \\
\hline 170 & 85 & 7 & 14 & 0.08 \\
\hline 171 & 19 & 2 & 18 & 0.11 \\
\hline 176 & 33 & 3 & 16 & 0.09 \\
\hline 182 & 169 & 13 & 14 & 0.08 \\
\hline 183 & 61 & 5 & 15 & 0.08 \\
\hline 183 & 183 & 14 & 14 & 0.08 \\
\hline 190 & 20 & 2 & 19 & 0.10 \\
\hline 190 & 76 & 6 & 15 & 0.08 \\
\hline 195 & 91 & 7 & 15 & 0.08 \\
\hline 196 & 49 & 4 & 16 & 0.08 \\
\hline
\end{tabular}




\section{Referências Bibliográficas}

COCHRAN, William G., COX, Gertrude M. Experimental Design. New York: John Wiley, 1957.

FEDERER, Walter Theodore. Experimental Design, Theory and Application. New York: Macmillan, 1955.

KEMPTHORNE, Oscar. Design and Analysis of Experiments. New York: Wiley \& Sons, 1975.

VAJDA, S. The Mathematics of Experimental Design: Incomplete Block Designs and Latin Squares. London: Griffin, 1967.

WINER, B. J. Statistical Principles in experimental Design. New York: McGraw-Hill, 1962.

YATES, Frank S. Experimental Design: Selected Papers of Frank Yates. London: Griffin, 1970. 